\title{
Photoemission Spectroscopy at Liquid Microbeams with a High Harmonics Table Top Radiation Source
}

\author{
Bernd Abel \\ University of Leipzig \\ Germany
}

\section{Introduction}

The use of ultrafast light pulses to visualize chemical dynamics has advanced our fundamental understanding of chemical and biochemical processes, by providing a means of monitoring the motion of atoms within a molecule in real time (Assmann et al. (2003)). However, closer to the chemist's heart is the evolution of the electron density in a molecule as molecules transform - as a function of time. In fact, the chemist's dream is to trace orbitals in time during a chemical transformation (Kling et al. (2006)). The most attractive medium in this context is the liquid phase where chemistry happens (Siefermann et al. (2010)). Recent progress in electronic spectroscopy in the condensed phase (e.g., Link, Lugovoy, Siefermann, Liu, Faubel \& Abel (2009) and Link, Voehringer-Martinez, Lugovoj, Liu, Siefermann, Faubel, Grubmueller, Gerber, Miller \& Abel (2009)) has been driven by the development of new ultrashort pulsed light sources, as well as the development of new experimental techniques. Recent successful experiments have made use of sources of ultrafast extreme-UV as probes of ultrafast molecular and materials dynamics (Paul et al. (2001)). These new probes can in principle directly provide site-specific molecular dynamics or local order via photoelectron spectroscopy, providing the potential to directly observe chemical reactions in atomic-level detail as they occur without the need for specific chromophors. The new tools appear to provide a powerful new window into the microscopic world, particularly as the temporal resolution and energy range of these sources continues to improve. One recent area of research motivated by these goals and the quest for fundamental light matter interactions is the generation of ultrashort EUV (extreme ultraviolet) light pulses through the process of high-order harmonic generation (HHG). HHG pushes traditional nonlinear optics to an extreme, by coherently combining many laser photons together to generate coherent beams that span from the UV to the keV-region of the spectrum (Krausz \& Ivanov (2009)). The pulses from HHG may be femtosecond to attosecond in duration. Thus, in the wavelength region, where these beams are bright and where they contain enough photons $(<100 \mathrm{eV})$, they have been used to monitor a variety of processes in chemical and materials science and for applications such as holographic imaging and photoelectron emission spectroscopy (Krausz \& Ivanov (2009), Kapteyn et al. (2007)).

As a liquid phase medium water has many unusual properties when compared with simple organic liquids (Head-Gordon \& Johnson (2005)), and bulk water as well as single water molecules play a decisive role in many chemical and biological systems. The dynamical network of hydrogen bondend water in chemical and biological systems has been studied 
with powerful spectroscopic techniques and theoretically in the past (Cowan et al. (2005), Fecko et al. (2003), Woutersen \& Bakker (2006), Head-Gordon \& Johnson (2005)).

Due to the technical and conceptual problems with (volatile) liquids in vacuum, liquid phase (high pressure) ESCA (electronic spectroscopy for chemical anylysis) is much less well established than XPS (x-ray photoelectron spectroscopy) at solid state surfaces (Siegbahn (1985), Lundholm et al. (1986)). Since Faubel et al. in Goettingen developed liquid beams in vacuum also volatile liquids like water could be investigated with photoelectron spectroscopy (Faubel \& Kisters (1989), Faubel (2000)) Since then, photoelectron emission lines and the chemical shift in the static ESCA (Winter \& Faubel (2006)), have also been particularly powerful observables for probing electron densities and molecular orbital energies in different intramolecular and intermolecular environments. Recently, we have combined powerful technologies such as photoelectron spectroscopy near volatile liquid interfaces in vacuum, ultrafast pump-probe spectroscopy, and table-top high-harmonics generation of soft X-ray radiation, which enabled us to perform liquid phase photoelectron spectroscopy with high time-resolution, i.e., adding the dimension of time to the liquid interface ESCA or liquid-phase XPS (Link, Lugovoy, Siefermann, Liu, Faubel \& Abel (2009) Link, Voehringer-Martinez, Lugovoj, Liu, Siefermann, Faubel, Grubmueller, Gerber, Miller \& Abel (2009)). The idea of ESCA combined with ultrafast EUV radiation has also been reported before (Drescher (2004)) but not for liquid phase matter. In the present chapter we will highlight and discuss, which insight this somewhat involved novel experimental approach can give us. As outlined above, it maps out a different kind of molecular information other than site specific chromophores or structural (diffraction) information - the orbital energy or electron density in a molecule in a special intramolecular and intermolecular environment. In the present contribution we aim to introduce into the new technology, highlight some recent results, and to show what is special and characteristic about time-resolved ESCA or XPS near liquid interfaces, in particular liquid water interfaces.

\section{Liquid beams in vacuum and high harmonics generation}

\subsection{Liquid water beam technology}

Liquid water jets in vacuum have been introduced by Faubel at the end of the 1980s (Faubel \& Kisters (1989)). A major obstacle in handling common liquids in vacuum is their large vapour pressures, which make it virtually impossible to maintain the required vacuum without either freezing the sample or having it consumed by evaporation and spoiling the vacuum. A liquid jet in vacuum including the nozzle assembly is displayed in Fig. 1. While the left figure shows the nozzle assembly the right part shows the details of the micro beam relevant for an experiment.

The enlarged section in Fig. 1 shows a high speed image of a beam with a 2-3 mm continuous section of the beam that decomposes into droplets (classical Rayleigh limit). The liquid jet is typically driven by a HPLC-pump at 10 to 50 bar at flow speed of $20-50 \mathrm{~m} / \mathrm{s}$ up to 100 $\mathrm{m} / \mathrm{s}$ (Charvat et al. (2004)). The temperature of the beam (surface) due to evaporative cooling can be calculated and predicted. It is plotted against the distance from the nozzle orifice in Fig. 2 for liquid water, ethanol, and methanol, respectively. More details about the liquid jet technology are given in Ref. (Charvat et al. (2004))

\subsection{High harmonics generation and their characteristics}

Harmonic generation is a nonlinear optical process in which the frequency of laser light is converted into its integer multiples. Odd harmonics of very high orders are generated due to symmetry reasons from noble gas atoms exposed to intense (usually near-infrared) laser fields. The spectrum from the process of high harmonic generation (HHG) typically consists 


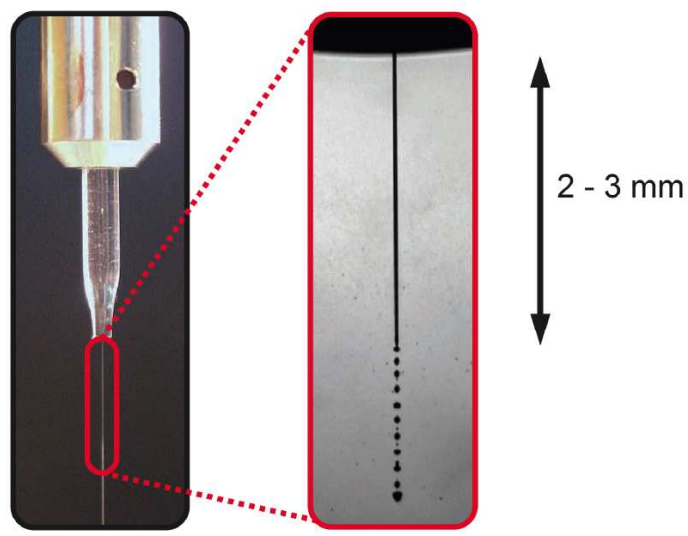

Fig. 1. Liquid beam (diameter $=15 \mu \mathrm{m}$ ) and nozzle assembly. The enlarged section displays the continuous section of the liquid beam and the decay into droplets behind the Rayleigh limit.

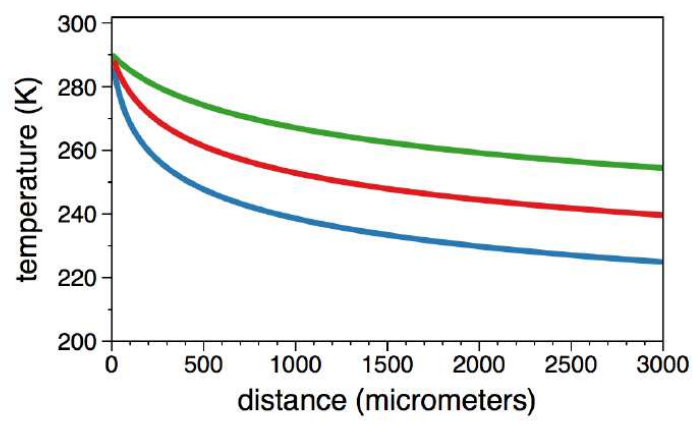

Fig. 2. Effect of evaporative cooling as a function of distance from the nozzle exit for a $17 \mu \mathrm{m}$ nozzple at $0.3 \mathrm{ml} / \mathrm{min}$ flow and a flow speed of the jet of about $22 \mathrm{~m} / \mathrm{s}$. Color code: green: water; red: ethanol; blue: methanol.

of a plateau, where the harmonic intensity is nearly constant over many orders of magnitude followed by a sharp cutoff. The maximal harmonic photon energy $\mathrm{E}_{c}=\mathrm{hv}$ is given by the well known cutoff law (Krause et al. (1992)),

$$
E_{c}=I_{p}+3.17 U_{p},
$$

where $\mathrm{I}_{p}$ is the ionization potential of the target atom, and $\mathrm{U}_{p}$ the ponderomotive energy, respectively (Seres et al. (2004), Seres et al. (2006)). HHG has now been established as one of the best methods to produce ultrashort coherent light pulses covering a wavelength range from the vacuum ultraviolet to the soft x-ray region (Attwood (2000)). The development of HHG has opened new research areas such as attosecond science (Krausz \& Ivanov 
(2009)) and nonlinear optics in the extreme ultraviolet (xuv) region. Many features of HHG can be intuitively and sometimes even semi-quantitatively explained in terms of electron re-scattering trajectories, which are described by the semiclassical three-step model (Corkum (1993)). Since the first demonstration of high-harmonic order generation in 1987, (femtosecond) laser-driven high-harmonic generation sources have become increasingly important for the generation of coherent extreme-ultraviolet radiation and soft x-rays down to the water window $(\lambda=4.4$ to $2.3 \mathrm{~nm}$ ) and up to the keV-regime (Seres et al. (2006), Seres et al. (2005)). Compared to synchrotrons and x-ray free electron lasers (XFELs), these sources are small-scale, much less expensive, and highly versatile, and their resulting unique characteristic output can be tailored more or less according to the experimental requirements (Hentschel et al. (2001)). Some drawback in comparison to the devices above is certainly the limited photon flux - the big advantage is their reliable availability in the laboratory. Ultrashort pulses of table top systems of reasonable brightness and high coherence are used in various experimental setups and applications ranging from atomic and molecular spectroscopy in the gas phase, liquid phase and solid-state surfaces, and, due to its coherent nature (Jaegle (2006)), harmonic emission is increasingly used for coherent-diffractive imaging (Attwood (2000), Kapteyn et al. (2007)).

The setup used in Goettingen for ultrafast photoelectron spectroscopy near the liquid water interface (microjet surface) with UV or IR pump and HHG-probe is illustrated schematically in Fig. 3. The inset displays high harmonics on a multi-channel-plate detector behind the grating for Neon (a) and Argon (b) as a HHG medium. Note, the HHG radiation travels in vacuum only. It is dispersed and focused with a toroidal grating. The time-resolution of a single grating setup is on the order of a few hundred femtoseconds, depending upon the HHG spot and the illuminated grooves on the EUV-grating. It can be improved by employing dielectric mirrors or a dual grating setup (Nugent-Glandorf et al. (2001)). Further details of the setup are given in (Link, Lugovoy, Siefermann, Liu, Faubel \& Abel (2009)).

\section{Towards ultrafast angle-resolved photoelectron spectroscopy at liquid interfaces}

In the ground state, the $\mathrm{H}_{2} \mathrm{O}$ molecule exhibits the electron configuration

$$
\left(1 a_{1}\right)^{2}\left(2 a_{1}\right)^{2}\left(1 b_{2}\right)^{2}\left(3 a_{1}\right)^{2}\left(1 b_{1}\right)^{2} .
$$

Here, $1 b_{1}, 3 a_{1}$ and $1 b_{2}$ are the three outer occupied molecular orbitals (MO) of water. The $1 b_{1}$ orbital is the highest occupied molecular orbital (HOMO) and its nodal plane lies in the plane including all atoms. The $1 b_{1}$ orbital has non-bonding character and hence is called a lone pair orbital. The $3 a_{1}$ and $1 b_{2}$ orbitals involve the $\mathrm{O}-\mathrm{H}$ bond, whereby the $1 b_{2}$ contributes the most. The $4 \mathrm{a}_{1}$ orbital is the lowest unoccupied molecular orbital (LUMO). In water aggregates, liquid water and ice, it accepts lone pair electrons from the $1 b_{1}$ orbitals of neighboring water molecules - in line with the definition of Pauling of hydrogen bonding.

The direction of photoelectron emission in the gas phase and near interfaces (liquid or solid) is intrinsically non-trivial and more or less anisotropic in general (Banna et al. (1986)). The differential photoemission cross section for a single electron orbital in free atoms and molecules is given by

$$
d \sigma / d \Omega=\sigma_{0} / 4 \pi\left(1+\beta P_{2}(\cos \theta)\right) .
$$

$\sigma_{0}$ defines the total photoelectron emission cross for this orbital, and, $\beta$ is called the anisotropy parameter. $\mathrm{P}_{2}(\cos \theta)$ is the second Legrende polynom (Attwood (2000)).

Up to the present, the anisotropy parameters $\beta$ and $\sigma$ for the molecular valence orbitals of water have only been measured for isolated gas phase water molecules, but not for liquid water. It has instead been assumed that they do not differ significantly from the values 


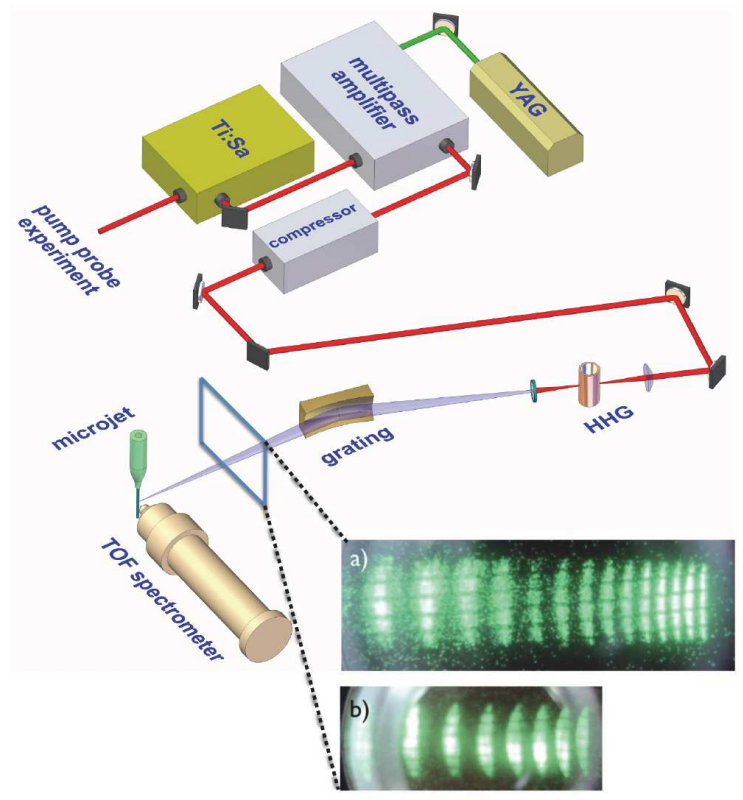

Fig. 3. Schematic view on the table top beam line experiment and the components for the photoelectron spectroscopy experiment. The essential units are depicted: Ti:Sa system, multipass amplifier, compressor, generation and selection of EUV light via HHG and toroidal grating. Inset: Generation of high harmonics of the fundamental $800 \mathrm{~nm}$ radiation in a neon or argon filled capillary. a) A sequence of high harmonics generated in neon, imaged by a XUV-sensitive CCD camera.The range of photon energies spans $40-70 \mathrm{eV} \mathrm{b}$ ) A sequence of high harmonics generated in argon. The range of photon energies in this case is between 25 and $40 \mathrm{eV}$. Note: it is only possible to generate the odd harmonics of the fundamental in both cases. Note: the EUV beam is in vacuum only up to the final chamber containing the liquid beam and the photoelectron spectrometer.

in the gase phase (Winter \& Faubel (2006)). This lack of data is due to the challenges of an appropriate experimental setup. For the investigation of the angular distribution of photoelectron emission, measurements of photoelectrons at different angles between the photoelectron detecting direction and the polarization vector of the ionizing radiation have to be made. The adjustment of the photoelectron emission angle can be realized by rotating either the axis of the spectrometer or the polarization vector of the incident light. If the spectrometer is fixed in the setup, a suitable radiation source is needed, which can be changed in the polarization. Although, possible in principle at beamlines at a few stations polarization resolved experiments are rare - and not done for liquid water. In a table-top HHG experiment the EUV radiation polarization can be readily controlled via control of the fundamental radiation polarization (Link, Lugovoy, Siefermann, Liu, Faubel \& Abel (2009)).

For the investigation of the angular distribution of emitted photoelectrons (at least) measurements at 3 different angles of polarization (HHG radiation) are necessary, if the spectrometer position is fixed. For this purpose, the polarization vector of the EUV light 


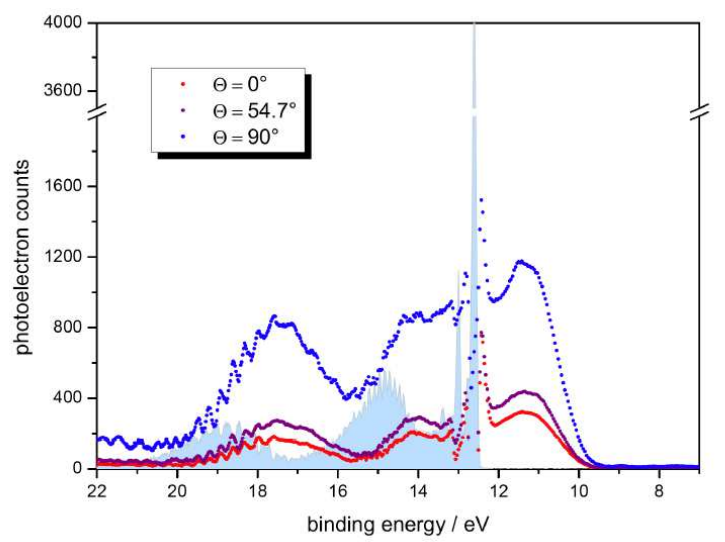

Fig. 4. Liquid beam photoelectron spectra at different angles. The photoelectron spectra are scaled to the $1 b_{1}$ gas phase peak intensity. The light blue spectrum ist he spectrum of water in the gas phase measured with p-polarized EUV light.

will be tuned via the polarization of the fundmantal, since the photoelectron analyzer, a time-of-flight photoelectron spectrometer, is fixed perpendicular to the direction of light propagation and in the plane of the optical table. The $\lambda / 2$ plate is positioned in the beam path of the fundamental radiation directed into the HHG chamber and in front of the iris that tailors the beam diameter of the $800 \mathrm{~nm}$ light. By rotating the optical axis of the $\lambda / 2$ plate the electron emission is measured at the required angle. The initial polarization plane of the $800 \mathrm{~nm}$ fundamental light is parallel to that of the optical table, on which the HHG chamber is mounted. At p-polarization, the angle $\theta$ between the photoelectron detecting direction and the polarization vector of the ionizing radiation is $0^{\circ}$, while $\theta=90^{\circ}$ for s-polarization. In addition, there is another relevant angle at $\theta=54.7^{\circ}$, also called the magic angle, which plays an important role in the characterization of the angular distribution of photoionization (Attwood (2000)). It must be noted that the focusing grating used here has different reflection properties for EUV radiation with various polarization vectors, which has been taken into account here.

The photoelectron emission spectra of liquid water for different angles of polarization of the high harmonics radiation are shown in Fig. 4. The spectra are normalized with respect to the $1 b_{1}$ gas phase photoelectron emission peak. The pure gas phase spectrum (blue shaded) has been measured with a p-polarization of the EUV beam. These spectra translate into the anisotropy diagram in Fig. 5. For a HHG energy of $38.7 \mathrm{eV}$ the anisotropy parameters $\beta$ and $\sigma$ of liquid in comparison to gas phase water for the differnt orbitals have been determined to be $1 b_{1}(\beta=0.8$ and $\sigma=1), 3 a_{1}(\beta=0.7$ and $\sigma=0.88)$, and $1 b_{2}(\beta=0.6$ and $\sigma=0.94)$ for liquid water, and $1 \mathrm{~b}_{1}(\beta=1.4$ and $\sigma=1), 3 \mathrm{a}_{1}(\beta=1.1$ and $\sigma=0.88)$, and $1 \mathrm{~b}_{2}(\beta=0.7$ and $\sigma=0.94)$ for gas phase water. The latter are close to those measured by Banna et al. some time ago (Banna et al. (1986)). What is important and obvious here is the observation that the $\beta$-values of liquid and gas phase water are significantly different, while the $\sigma$-values are quite similar.

\section{Hydrogen bonding in extreme states of water}

If very short infrared radiation pulses tuned to a strong $\mathrm{OH}$-stretch vibration absorption of water around $3 \mu \mathrm{m}$ are used, it is possible to heat the water extremely, at a rate faster than the 


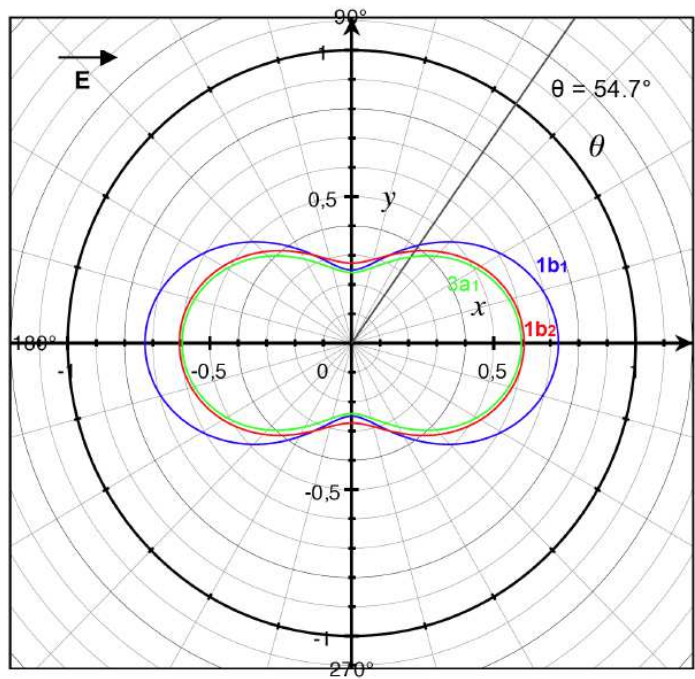

Fig. 5. Angular distribution of photoelectrons from the $1 b_{1}$ (blue), $3 a_{1}$ (green) and $1 b_{2}$ (red) orbitals of liquid water. The ionizing photon energy is $38.7 \mathrm{eV}$. These curves are based on our experimentally obtained $\beta$ and $\sigma$-values and assuming that the $\sigma$ ?-values are similar and do do not change significantly for water molecules in different aggregate states.

thermal expansion rate and to prepare extreme states of water (Link, Lugovoy, Siefermann, Liu, Faubel \& Abel (2009), Link, Voehringer-Martinez, Lugovoj, Liu, Siefermann, Faubel, Grubmueller, Gerber, Miller \& Abel (2009)). These states can have temperatures well above the boiling point and it has been shown that the water phase may be even heated significantly above the critical temperature (Debenedetti (1996)). We have recently investigated metastable, superheated and supercritical phases with time-resolved photoelectron spectroscopy with IR excitation and EUV probe. As shown in Ref. (Link, Voehringer-Martinez, Lugovoj, Liu, Siefermann, Faubel, Grubmueller, Gerber, Miller \& Abel (2009)) the initial temperature can be estimated via the known absorption coefficients, penetrations depth of the IR beam into the water phase, and the laser focus diameter, after laser induced heating. If water is prepared at these extreme states, it is known that it literally explodes at the extreme conditions, however, at the femtosecond or picosecond time-scale it moves slowly in time and can thus be resolved with ultrafast spectroscopy. Different than with other spectroscopies time-resolved photoelectron spectra in the time-domain measure the chemical shift of the valence electrons of water in time, which provide interesting information about the question how the phase and the hydrogen-bonding network 'look like' and how they evolve in time. In a recent contribution we reported investigations on the molecular photoemission signature of the evolution of the phase and the hydrogen-bonding network of super-heated water below and above the critical point, as well as its timescales. Typical results are displayed in Fig. 6. In the plots the binding energy (being the difference of the photon energy and the kinetic energy of the photoelectrons measured in the experiment via a time-of-flight electron spectrometer, $\left.E_{b}=E_{p h}-E_{k i n}\right)$ is plotted against the photoelectron emission counts resulting in a typical photoelectron spectrum in Fig. 6. In a typical valence electron photoemission spectrum of water the gas phase emission lines of the $1 b_{1}, 3 a_{1}$, and $1 b_{2}$ can be assigned easily (Winter et al. 
(2004)). The corresponding lines of the orbitals in the liquid phase are shifted toward lower binding energies but they are significantly broader and overlapping. Since the corresponding $1 b_{1}^{\prime}$ orbital energy in the liquid phase is shifted most in the liquid phase it can be identified easily. A transition of water from the liquid phase to the gas phase is accompanied with a decrease in intensity in the most shifted $1 b_{1}^{\prime}$ emission line (color coded in red) of liquid water.

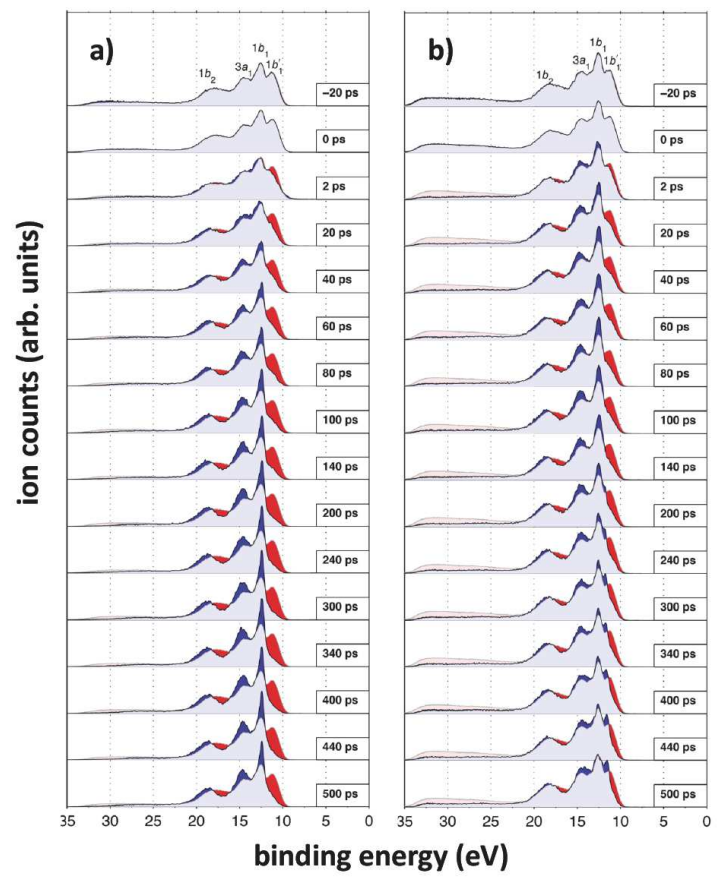

Fig. 6. Time-resolved photoelectron spectra of liquid (before excitation at neg. delay time) and metastable water (left: excitation at $\lambda_{I R}=2650 \mathrm{~nm}$; right panel: $\lambda_{I R}=2830 \mathrm{~nm}$ :). For further details see the text and Refs. (Link, Lugovoy, Siefermann, Liu, Faubel \& Abel (2009), Link, Voehringer-Martinez, Lugovoj, Liu, Siefermann, Faubel, Grubmueller, Gerber, Miller \& Abel (2009)).

At the same time an increase in intensity of the gas phase lines $\left(1 b_{1}, 3 a_{1}\right.$, and $\left.1 b_{2}\right)$ is observable (color coded in blue in Fig. 6). Different hot phases are correlated with the overall time-depedence of the signals and the dispersion of the liquid phase, which is observable in the binding energy region between the liquid phase $1 b_{1}^{\prime}$ peak and the coresponding gas phase peak. This is typically the range in which photoemission of more of less large clusters have been observed (see Refs in Link, Lugovoy, Siefermann, Liu, Faubel \& Abel (2009) and Link, Voehringer-Martinez, Lugovoj, Liu, Siefermann, Faubel, Grubmueller, Gerber, Miller \& Abel (2009)). In particular, the hotter phase in Fig. 6 (right panel) displays a higher degree of dispersion into smaller clusters. The overall chemical shift of the $1 b_{1}^{\prime}$ of the superheated phase towards larger binding energies is noticeable but small. In Ref. (Link, Voehringer-Martinez, Lugovoj, Liu, Siefermann, Faubel, Grubmueller, Gerber, Miller \& Abel (2009)) we have shown that quantitative photoelectron emission detection and evaluation through a hot and moving interface is possible and that the observation depth is a few monolayers of water and alcohol 
molecules at the interface (Itikawa \& Mason (2005)). The dynamics of superheated water and alcohols at the liquid-vacuum interface has been compared with large-scale molecular dynamics calculations that capture the dynamics of the solvents near their liquid and super heated interface. It is not unexpected that the dynamics of the phase evolution after laser heating is governed by the highly dynamical evolution of the network of hydrogen bonds (Link, Voehringer-Martinez, Lugovoj, Liu, Siefermann, Faubel, Grubmueller, Gerber, Miller \& Abel (2009)).

\section{Ultrafast concerted electron motion of a plasma at a liquid water surface}

Various methods have been applied to investigate the parameters of laser-induced plasmas (see Ref. (Link, Lugovoy, Siefermann, Liu, Faubel \& Abel (2009)) and refs therein). A plasma near a water interface constitutes a special case of coherent electron motion that can be observed with ultrafast photoelectron spectroscopy. Using optical near IR fs-pulses a surface plasma can be generated through multi photon absortion and ionization. If it is formed near an exploding liquid beam in vacuum the plasma first oscillates at the plasma frequency and then decays due to the overall decay of the hot water filament. High harmonics radiation can be employed to induce electron emission from the valence bands of dense water at the interface and gas phase water, which should be sensitive to the local plasma conditions. Thus we have anticipated that a plasma can be investigated with the new EUV photoelectron spectroscopy. In Ref. (Link, Lugovoy, Siefermann, Liu, Faubel \& Abel (2009)) we report first experiments in which we have investigated the dynamics, i.e., the oscillation and decay of a plasma near a liquid water jet, as well as some of its characteristic parameters. IR pulses at an energy of of 20-30 $\mu \mathrm{J}$ at $2650 \mathrm{~nm}$ were employed to trigger and induce the plasma. For pulse durations of $100 \mathrm{fs}$ and a focus diameter of $80-100 \mu \mathrm{m}$ a peak intensity of well above $10^{12} \mathrm{~W} / \mathrm{cm}^{2}$ is reached, which is sufficient to induce a plasma. The 25th harmonic of $800 \mathrm{~nm}$ radiation at $38.6 \mathrm{eV}$ photon energy was chosen for the probe, i.e., the photoelectron emission from the valence bands of water. In the vicinity of a liquid beam in vacuum a significant but low vapor pressure was documented (Faubel et al. (1997)). In Fig. 7 (apparently) periodically changing 'binding energies' and photoelectron spectra as a function of time of a near liquid interface plasma, that displays distinct oscillations, is depicted (Link, Lugovoy, Siefermann, Liu, Faubel \& Abel (2009)). The characteristics of the oscillating 'binding energies' suggest that the electrons stem from and belong to the water molecules, however, they are not tightly bound anymore to the water in the gas phase, but both are part of an ensemble of ionized cations and electrons oscillating coherently in time. The plot also shows photoelectrons with large kinetic energies close to the photon energy.

At early times the IR pump pulse initiates plasma formation near the liquid beam interface (a number of 22 photons at $2650 \mathrm{~nm}$ would be necessary to result in an ionization energy of $10 \mathrm{eV}$ ). In Ref. (Link, Lugovoy, Siefermann, Liu, Faubel \& Abel (2009)) we have determined a period of oscillation of major photoemission lines of $1.5 \mathrm{ps}$ corresponds to a plasma frequency of $0.67 \mathrm{THz}$ and a free electron density close to of $5.6 \times 10^{15} \mathrm{~cm}^{-3}$. This is a quite low level of electron density. It is worth noting that with this setup we have realized a pulsed ultrafast THz-Oscillator that should emit pulsed picosecond THz-radiation (not measured in (Link, Lugovoy, Siefermann, Liu, Faubel \& Abel (2009))). At $2650 \mathrm{~nm}$ the resonance frequency of a laser driven plasma is $113 \mathrm{THz}$, which corresponds to a free electron density of 1.6 $x 10^{20} \mathrm{~cm}^{-3}$. This number is more than one order of magnitude lower than the critical resonance free electron density for $800 \mathrm{~nm}$ radiation. The time scale of the decay of the plasma in the few picoseconds regime is related to the transport of hot water molecules in the expanding (exploding) beam interface, which has been estimated from molecular dynamics simulations. This is again a nice example for the power of the pump-EUV-photoelectron 


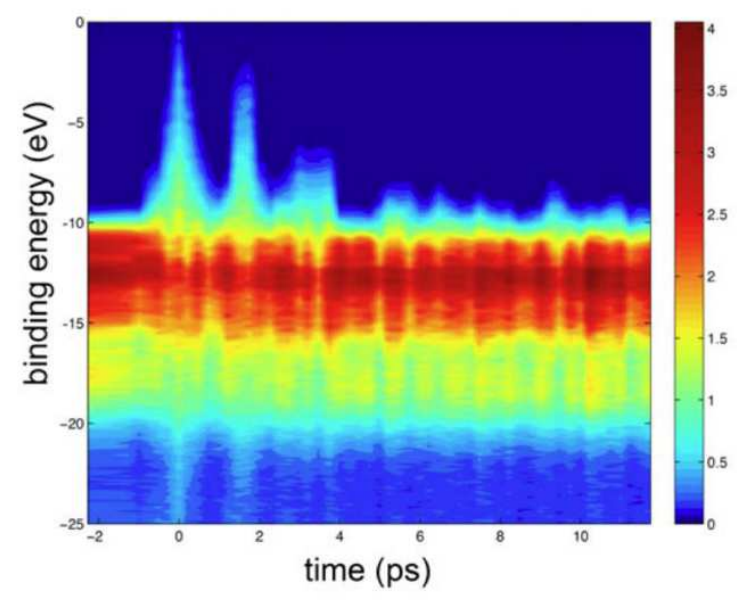

Fig. 7. Kinetic energies of photoelectrons as a function of time on top of the water photoelectron spectrum (spectra represent a vertical cut) in the case of an oscillating near liquid water surface plasma. An oscillation period of the fast electrons of $1.5 \mathrm{ps}$ corresponding to a plasma frequency of $0.67 \mathrm{THz}$ and a free electron density of $6 \times 10^{15} \mathrm{~cm}^{-3}$ (for more details see the text).

emission probe technology near volatile liquid interfaces enabling real-time probing of laser-ionized plasmas with high temporal resolution, yielding information that is hardly accessible by other methods.

\section{The solvated electron in water - news from a seemingly well known transient}

Further development of liquid phase time-resolved photoelectron spectroscopy in our lab made it possible to directly measure the vertical binding energy (VBE) of one of the most important chemical transients in aqueous solution: the solvated electron in liquid water (Boag \& Hart (1963)), which is also termed hydrated electron or $e^{-}(a q)$ (Siefermann et al. (2010)). In the most common picture, a hydrated electron is located in a non-spherical cavity formed by about 6 water molecules (Kevan (1981)). The VBE corresponds to the energy, which is required to completely remove the electron from this cavity, without changing the initial geometry of the system. The VBE is a crucial quantity for understanding the reactivity of hydrated electrons with DNA bases in DNA damaging processes - or many other electron transfer and attachment processes (Sanche (2009), Lu (2009)). Shortly after the report by Siefermann et al. in 2010 three more publications confirmed the binding energy of the bulk hydrated electron (Tang et al. (2010), Shreve et al. (2010), Luebcke et al. (2010)).

For a long time researchers tried to estimate the binding energy of hydrated electrons from data of large anionic water clusters (Neumark (2008)). In 2010, Siefermann et al. for the first time directly measured the vertical binding energies of $e^{-}(a q)(\mathrm{VBE}=3.3 \pm 0.1 \mathrm{eV})$ and of electrons solvated/hydrated at the water surface $e^{-}(s f)(\mathrm{VBE}=1.6 \pm 0.1 \mathrm{eV})$ using the experimental pump-probe setup employing UV pump- and EUV-probe pulses (see Fig. 3 ). The key feature of the experimental approach is the generation of solvated electrons by short pump pulses of $267 \mathrm{~nm}$ light (Chen \& Bradforth (2008)) and recording photoelectron spectra at the same time employing time-delayed $38.7 \mathrm{eV}(32 \mathrm{~nm})$ high harmonic probe 

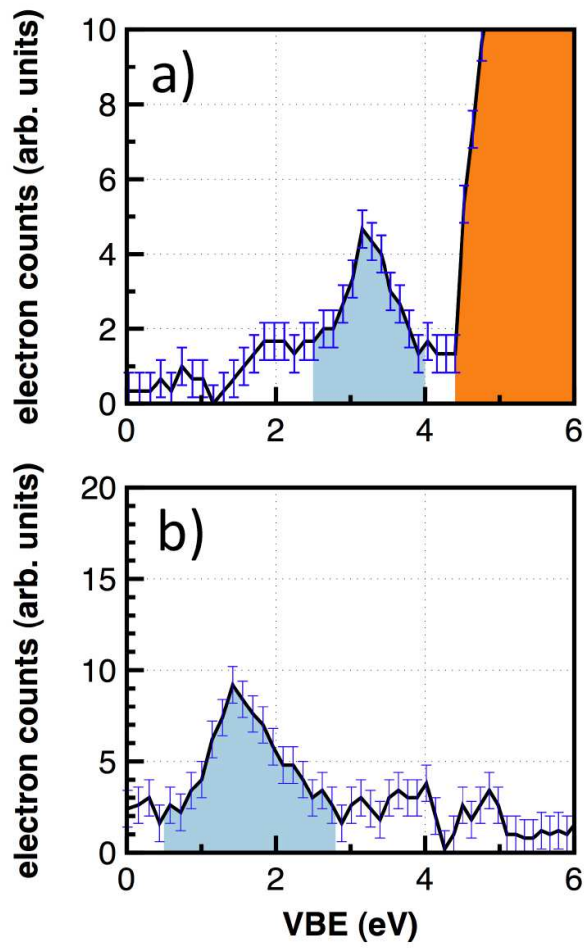

Fig. 8. Photoelectron spectrum and binding energy of the fully hydrated electron (a) and the surface hydrated electron (b) near the liquid water interface. While the first transient is prepared via a one photon excitation (low laser light intensity) of $\mathrm{K}_{4}\left(\mathrm{Fe}(\mathrm{CN})_{6}\right)$ in water the latter is prepared via two-photon excitation (high laser light intensity) of water at $270 \mathrm{~nm}$. The emission bands from both hydrated electron binding motifs exist only if a $270 \mathrm{~nm}$ excitation pulse precedes the EUV-probe (both colored in blue). The brown emission band stems from the iron ion $\left(\mathrm{Fe}^{2+}\right)$ in the precursor complex. The noise level is indicated.

pulses. In order to access the properties of $e^{-}(s f)$, pure water was ionized via the two-photon absorption of $267 \mathrm{~nm}$ light, which results in the formation of bulk $\left(e^{-}(a q)\right)$ and surface solvated $\left(e^{-}(s f)\right)$ electrons (Chen \& Bradforth (2008)). When subsequently recording the photoelectron spectrum with the $38.7 \mathrm{eV}$ probe pulse, the detection efficiency is highest for photoelectrons originating from the surface, and declines dramatically with every layer of water molecules (Ottosson et al. (2010)). The reason lies in the mean free path of photoelectrons, which is particularly short (Attwood (2000)) for the resulting photoelectron kinetic energies of about $35 \mathrm{eV}$ (38.7 eV - VBE). This allowed us to directly measure properties of $e^{-}(s f)$, and it demonstrates that our experimental setup is particularly suited for the investigation of transient surface species, such as $e^{-}(s f)$.

In order to access the VBE of $e^{-}(a q)$ with our experimental setup, it is required to significantly increase the concentration of $e^{-}(a q)$, and at the same time suppress the formation of $e^{-}(s f)$. This 'inversion' type of situation is realized by employing $\left[\mathrm{Fe}(\mathrm{CN})_{6}\right]^{4-}$ complexes in aqueous solution as precursors, which primarily yield $e^{-}(a q)$ after the absorption of one 267nm 
photon and which are literally repelled from the water surface (Siefermann et al. (2010)). Suppressing two-photon processes by lowering the $267 \mathrm{~nm}$ pump pulse intensity inhibits the formation of $e^{-}(s f)$ and allows for recording the signature of the fully hydrated electron $e^{-}(a q)$. Figure 8 presents the photoelectron spectra of $e^{-}(a q)(\mathrm{a})$ and $e^{-}(s f)(\mathrm{b})$. Evaluation of the relative signal heights reveals that signal intensities both species (binding motifs) are on the same order of magnitude. For different pump-probe time-delays between 7 ps and 100 ps, no significant changes in the photoelectron spectrum of $e^{-}(s f)$ were observed, which demonstrates that the lifetime of $e^{-}(s f)$ exceeds $100 \mathrm{ps,} \mathrm{which} \mathrm{was} \mathrm{tentatively} \mathrm{attributed} \mathrm{to}$ a dynamic barrier that stems from rearranging hydrogen bonds (Siefermann et al. (2010)). Follow-up experiments by a number of other groups employing UV light (267 - $213 \mathrm{~nm})$ to probe the binding energy of the bulk hydrated electron yielded values of $3.27 \mathrm{eV}$ (Tang et al. (2010)), $3.6 \mathrm{eV}$ (Shreve et al. (2010)), and $3.4 \mathrm{eV}$ (Luebcke et al. (2010)), which are close to the value initially reported in Ref. (Siefermann et al. (2010)).

\section{Conclusions and outlook}

In summary, this work demonstrates the potential for a new kind of ultrafast liquid phase spectroscopy, based on high-harmonics generation and liquid microbeam technology, that has unique advantages. First and foremost, the technique is very surface sensitive at wavelength in the extreme UV. This changes for shorter wavelengths. In particular, wavelength tuning should enable depth profiling from the liquid interface to the bulk - a very intruiging possibily. Time-resolution can be increased routinely nowadays (with commercial products) to a few femtoseconds enabling a new time window for molecular dynamics. This time window is reduced by recent efforts to produce stable single attosecond pulses in the extreme UV spectral range. Several groups demonstrated the generation of attosecond light pulses using high harmonic generation and investigated processes on the attosecond to few femtosecond timescale. One of our near future goals is it to improve the time-resolution of our experimental setup towards the few femtosecond timescale. The quest for higher and higher harmonics and shorter wavelength also enables new avenues to perform real ESCA experiments in the water window at around 3-4 nm. This is very close to the heart of a chemist, which enables us to watch chemical reactions though their change in electron density and orbital evolution as they happen in solution. Furthermore, the high harmonic probe should not be limited by 'dark states' present in other spectroscopies. High harmonics radiation can be employed as an in situ and local and site-specific probe of femtosecond and even attosecond nuclear and electron dynamics in molecules surrounded by a solvent or dense environment. Finally, the table top beamline setup is a very valuable tool to pre-study systems and optimal conditions for experiments at synchrotron beam limes such as the free electron lasers (XFEL, FLASH, LCLS) in Hamburg and Stanford, which will have superior performance but very limited beam time. To just repeat an experiment performed with somewhat limited laser driven table top systems with optimal conditions at a large scale x-ray laser facility is much more rewarding and promising than optimizing experiments on site. Therefore, experimental setups highlighted here will have a very bright future, even though large scale $x$-ray laser facilities are being build and ready to use in the near future.

\section{Acknowledgement}

This work has been generously supported by the Deutsche Forschungsgemeinschaft though the SPP1134 (Investigation of Transient Structures of Photo-Induced Chemical Reactions in Solution with Ultrafast XUV-Photoelectron Spectroscopy), the SFB 755 (Nanoscale Photonic Imaging), the Graduate School 782 (Dynamics and spectroscopy of molecular aggregates, coils and networks), the Fonds der Chemischen Industrie, as well as the Volkswagenstiftung. 
Contributions of Dr. O. Link, Dr. K. Siefermann, Dr. Y. Liu, Dr. E. Lugovoy, and Dr. M. Faubel to the work and technology described here are gratefully acknowledged. The author also thanks M. Faubel, B. Winter, U. Buck, and E. Lugovoy for stimulating discussions on this topic.

\section{References}

Assmann, J., Kling, M. \& Abel, B. (2003). Watching photo induced chemistry and molecular energy flow in solution in real-time, Angewandte Chem Int. Ed. 42: 2226-2246.

Attwood, D. (2000). Soft X-Rays and Extreme Ultraviolet Radiation, Cambridge University Press.

Banna, M. S., McQuaide, B. H., Malutzki, R. \& Schmidt, V. J. (1986). The photoelectron spectrum of water in the 30-140 ev photon energy range, J. Chem. Phys. 84: 4739-4744.

Boag, J. W. \& Hart, E. J. (1963). Absorption spectra in irradiated water and some solutions absorption spectra of hydrated electron, Nature 197(486): 45.

Charvat, A., Lugovoy, E., Faubel, M. \& Abel, B. (2004). New design for a time-of-flight mass spectrometer, Rev.. Sci. Instr. 75: 1209-1218.

Chen, X. \& Bradforth, S. E. (2008). The ultrafast dynamics of photodetachment, Annu. Rev. Phys. Chem. 59: 203.

Corkum, P. B. (1993). Plasma perspective on strong-field multiphoton ionization, Phys. Rev. Lett. 71(13): 1994.

Cowan, M. L., Bruner, B. D., Huse, N., Dwyer, J. R., Chugh, B., Nibbering, E. T. \& Elsaesser, T. (2005). Ultrafast memory loss and energy redistribution in the hydrogen bond network of liquid h2o, Nature 434: 199-202.

Debenedetti, P. G. (1996). Metastable Liquids: Concepts and Principles, Academic Press, Princeton, New Jersey.

Drescher, M. (2004). Time-resolved esca: a novel probe for chemical analysis, Z.Phys. Chem. 218: $1147-1168$.

Faubel, M. (2000). Photoionization and Photodetachment, C. Y. Ng, World Scientific.

Faubel, M. \& Kisters, T. (1989). Non-equilibrium molecular evaporation of carboxylic acid dimers, Nature 339(6225): 527.

Faubel, M., Steiner, B. \& Toennies, J. P. (1997). Photoelectron spectroscopy of liquid water, some alcohols, and pure nonane in free micro jets, J. Chem. Phys. 106: 9013.

Fecko, C. J., Eaves, J. D., Loparo, J. J., Tokmakoff, A. \& Geissler, P. L. (2003). Ultrafast hydrogen-bond dynamics in the infrared spectroscopy of water, Science 301: 1698-1702.

Head-Gordon, T. \& Johnson, M. E. (2005). Tetrahedral structure or chains for liquid water, PNAS 103: 7973-7977.

Hentschel, M., Kienberger, R., Spielmann, C., Reider, G. A., Milosevic, N., Brabec, T., Corkum, P., Heinzmann, U., Drescher, M. \& Krausz, F. (2001). Attosecond metrology, Nature 414: 509-513.

Itikawa, Y. \& Mason, N. (2005). Cross sections for electron collisions with water molecules, J. Phys. Chem. Ref. Data 34: 1-22.

Jaegle, P. (2006). Coherent Sources of XUV Radiation, Springer-New York.

Kapteyn, H., Cohen, O., Christov, I. \& Murnane, M. (2007). Harnessing attosecond science in the quest for coherent x-rays, Science 317: 775-778.

Kevan, L. (1981). Solvated electron structure in glassy matrices, Acc. Chem. Res. 14: 138. Kevan structure of hydrated electron.

Kling, M. F., Siedschlag, C., Verhoef, A. J., Khan, J. I., Schultze, M., Uphues, T., Ni, Y., Uiberacker, M., Drescher, M., Krausz, F. \& Vrakking, M. J. J. (2006). Control of electron localization in molecular dissociation, Science 312(5771): 246. 
Krause, J. L., Schafer, K. J. \& Kulander, K. C. (1992). High-order harmonic generation from atoms and ions in the high intensity regime, Phys. Rev. Lett. 68: 3535.

Krausz, F. \& Ivanov, M. (2009). Attosecond physics, Rev. Mod. Phys. 81(1): 163.

Link, O., Lugovoy, E., Siefermann, K. R., Liu, Y., Faubel, M. \& Abel, B. (2009). Ultrafast electronic spectroscopy for chemical analysis near liquid water interfaces: concepts and applications, Appl. Phys. A 96(1): 117.

Link, O., Voehringer-Martinez, E., Lugovoj, E., Liu, Y., Siefermann, K., Faubel, M., Grubmueller, H., Gerber, R. B., Miller, Y. \& Abel, B. (2009). Ultrafast phase transition in metastable water near liquid interfaces, Faraday Discuss. 141: 67.

Lu, Q.-B. (2009). Correlation between cosmic rays and ozone depletion, Phys. Rev. Lett. 102(11): 118501.

Luebcke, A., Buchner, F., Heine, N., Hertel, I. \& Schultz, T. (2010). Time-resolved photoelectron spectroscopy of solvated electrons in aqueous nai solution, PCCP 12: 14692.

Lundholm, M., Siegbahn, H., Holmberg, S. \& Arbman, M. (1986). Core electron spectroscopy of water solutions, J. Electron. Spectrosc. Relat. Phenom. 40(2): 163.

Neumark, D. (2008). Spectroscopy and dynamics of excess electrons in clusters, Mol. Phys. 106: 2183.

Nugent-Glandorf, L., Scheer, M., Samuels, D. A., Mulhisen, A. M., Grant, E. R., Yang, X., Bierbaum, V. M. \& Leone, S. R. (2001). Phys. Rev. Lett. 87: 193002.

Ottosson, N., Faubel, M., Bradforth, S. E., Jungwirth, P. \& Winter, B. (2010). Photoelectron spectroscopy of liquid water and aqueous solution: Electron effective attenuation lengths and emission-angle anisotropy, J. Electron. Spectrosc. Relat. Phenom. 177(2-3): 60.

Paul, P. M., Toma, E. S., Breger, P., Mullot, G., Auge, F., Balcou, P., Muller, H. G. \& Agostini, P. (2001). Observation of a train of attosecond pulses from high harmonic generation, Science 292: 1689.

Sanche, L. (2009). Beyond radical thinking, Nature 461: 358-359.

Seres, E., Seres, J., Krausz, F. \& Spielmann, C. (2004). Phys. Rev. Lett. 92: 163002.

Seres, J., Seres, E., Verhoef, A. J., Tempea, G., Streli, C., Wobrauschek, P., Yakovlev, V., Scrinzi, A., Spielmann, C. \& Krausz, F. (2005). Source of coherent kiloelectronvolt x-rays, Nature 433: 596.

Seres, J., Wobrauschek, P., Streli, C., Yakovlev, V. S., Seres, E., Krausz, F. \& Spielmann, C. (2006). New J. Phys. 8: 251.

Shreve, A. T., Yen, T. A. \& Neumark, D. M. (2010). Photoelectron spectroscopy of hydrated electrons, Chem. Phys. Lett. 493: 216.

Siefermann, K. R., Liu, Y., Lugovoy, E., Link, O., Faubel, M., Buck, U., Winter, B. \& Abel, B. (2010). Binding energies, lifetimes and implications of bulk and interface solvated electrons in water, Nat. Chem. 2(4): 274.

Siegbahn, H. (1985). Electron spectroscopy for chemical analysis of liquids and solutions, J. Phys. Chem. 89: 897.

Tang, Y., Shen, H., Sekiguchi, K., Kurahashi, N., Mizuno, T., Suzuki, Y.-I. \& Suzuki, T. (2010). Direct measurement of vertical binding energy of a hydrated electron, PCCP 12: 3653.

Winter, B. \& Faubel, M. (2006). Photoemission from liquid aqueous solutions, Chem. Rev. 106: 1176.

Winter, B., Weber, R., Widdra, W., Dittmar, M., Faubel, M. \& Hertel, I. V. (2004). Full valence band photoemission from liquid water using euv synchrotron radiation, J. Phys. Chem. A 108(14): 2625.

Woutersen, S. \& Bakker, H. J. (2006). Ultrafast vibrational and structural dynamics of the proton in liquid water, Phys. Rev. Lett. 96: 138305. 


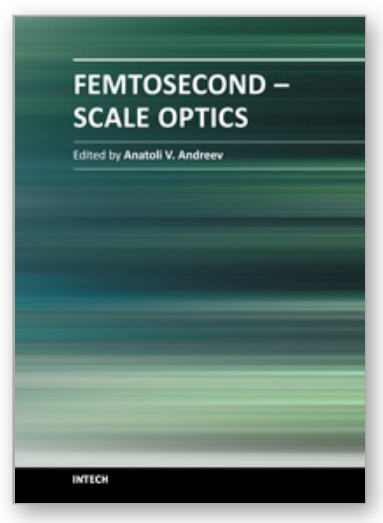

\author{
Femtosecond-Scale Optics \\ Edited by Prof. Anatoly Andreev
}

ISBN 978-953-307-769-7

Hard cover, 434 pages

Publisher InTech

Published online 14, November, 2011

Published in print edition November, 2011

With progress in ultrashort ultraintense laser technologies the peak power of a laser pulse increases year by year. These new instruments accessible to a large community of researchers revolutionized experiments in nonlinear optics because when laser pulse intensity exceeds or even approaches intra-atomic field strength the new physical picture of light-matter interaction appears. Laser radiation is efficiently transformed into fluxes of charged or neutral particles and the very wide band of electromagnetic emission (from THz up to $\mathrm{x}$-rays) is observed. The traditional phenomena of nonlinear optics as harmonic generation, self-focusing, ionization, etc, demonstrate the drastically different dependency on the laser pulse intensity in contrast the well known rules. This field of researches is in rapid progress now. The presented papers provide a description of recent developments and original results obtained by authors in some specific areas of this very wide scientific field. We hope that the Volume will be of interest for those specialized in the subject of laser-matter interactions.

\title{
How to reference
}

In order to correctly reference this scholarly work, feel free to copy and paste the following:

Bernd Abel (2011). Photoemission Spectroscopy at Liquid Microbeams with a High Harmonics Table top Radiation Source, Femtosecond-Scale Optics, Prof. Anatoly Andreev (Ed.), ISBN: 978-953-307-769-7, InTech, Available from: http://www.intechopen.com/books/femtosecond-scale-optics/photoemission-spectroscopy-atliquid-microbeams-with-a-high-harmonics-table-top-radiation-source

\section{INTECH}

open science | open minds

\section{InTech Europe}

University Campus STeP Ri

Slavka Krautzeka 83/A

51000 Rijeka, Croatia

Phone: +385 (51) 770447

Fax: +385 (51) 686166

www.intechopen.com

\section{InTech China}

Unit 405, Office Block, Hotel Equatorial Shanghai

No.65, Yan An Road (West), Shanghai, 200040, China

中国上海市延安西路65号上海国际贵都大饭店办公楼405单元

Phone: +86-21-62489820

Fax: $+86-21-62489821$ 
(C) 2011 The Author(s). Licensee IntechOpen. This is an open access article distributed under the terms of the Creative Commons Attribution 3.0 License, which permits unrestricted use, distribution, and reproduction in any medium, provided the original work is properly cited. 